\title{
ENERGY AND ANGULAR MOMENTUM DEPOSITION DURING COMMON ENVELOPE EVOLUTION
}

\author{
Noam Soker ${ }^{1,2}$ \\ RESUMEN
}

Tomo en consideración tres procesos que incrementan la tasa de pérdida de masa de la envolvente común de una gigante de la secucucia principal o de una compañera enana blanca que se precipita en espiral dentro de su envolvente. C'onsidero el depósito de energía orbital y momento angular orbital en la envolvente de la gigante y la formación de "jets" por la compañera acrecionte que se propagan dentro de la envolventc. Encuentro que en muchos casos el depósito del momento angular enl la chvolvente puede ser más importante para el proceso de pérdida de masa que el clepósito de encrgía orbital. En etapas tempranas de la evolución común de la envolvente los "jets" que son expulsados por una compañera acreciente, en particular una enana blanca que orbita dentro de liss regioness exteriores do la cuvolvente de la gigante, también pueden dominar sobre el depósito de energía. Esto implica que los estudios que ignoran el depósito del momento angular dentro de la envolvente y los efectos de la compañera acreciente pueden llegar a conclusiones erróneas.

\section{ABSTRACT}

I consider three processes which enhance mass loss rate from a common envelope of a giant star with a main sequence or a white dwarf companion spiraling-in inside its envelope. I consider deposition of orbital energy and orbital angular momentum to the giant's envelope, and the formation of jets by an accreting companion and their propagation in the envelope. I find that in many cases the deposition of orbital angular momentum to the envelope may be more important to the mass loss process than the deposition of orbital energy. Jets blown by an accreting companion, in particular a white dwarf. orbiting inside the outer regions of the giant's envelope may also dominate over orbital energy deposition at early stage of the common envelope evolution. These imply that studies which ignore the deposition of angular momentum to the envelope and the effects of the accreting companion niay reach wrong conclusions.

\section{Key Words: ISM: JETS AND OUTFLOWS - STARS: AGB AND POST-AGB - STARS: BINARIES - STARS: EVOLUTION - STARS: MASS LOSS}

\section{INTRODUCTION}

As a star in a binary system swolls to become a giant it engulfs its companion if the orbital separation is smaller than some critical value and if the companion is not too massive: a common envelope (CE) phase commences. (for a review sec Iben \& Livio 1993, and Taan \& Sandquist 2000 ). Because of tidal interaction and friction. the orbit shrinks. Several parameters ('an be defined to characterized the CE evolution (e.g. Livio (f Soker 1988). but the most commonly used parameter is the ratio of the orbital energy that is released during the CE phase $\Delta E_{i, r l}$, to the binding encrgy of the ejected envelope $\Delta E_{\text {bind }}:$ r $_{C E} \equiv \Delta E_{\text {bind }} / \Delta E_{\text {orl }}$. Noto that different definitions for the binding energy exist. (e.g., O'Brien, Bond. \& Sion 2001). Since the orbital energy that is relwised depends mostly on the final or-

\footnotetext{
'Department of Physics. Technion Israel Institute of Technology, Israel

${ }^{2}$ I eppartment of Physics. Oranim. Israel
}

bital separation, the value of $\alpha_{\mathrm{CE}}$ can be in principle calculated for systems whose final orbital separation is known, assuming the giant structure at the onset of the CE is known (O'Brien et al. 2001; Maxted et al. 2002). The use of the $\alpha_{\mathrm{CE}}$ is common also in numerical simulations of the CE phase (e.g. Sandquist, Taam \& Burkert 2000 for a recent paper). However, numerical simulations can't include the effect of enhanced mass loss rate from giant stars that have a very high mass loss rate. The spun-up envelope of red giant branch (RGB) and asymptotic giant branch (AGB) stars may have a much higher mass loss rate, with the energy source being the giant's luminosity rather than the orbital energy (Soker \& Harpaz 2003).

In some systems the usage of the above expression in a simple manner yields $\alpha_{\mathrm{CE}}>1$. For example, Maxted ot al. (2002) assume that negligible mass has been lost prior to the onset of the CE phase in PG1115+166, and find $\alpha_{\mathrm{CE}}>1$. This led some 
researchers to argue that the energy stored in the envelope, and in particular the ionization energy, i.e., the energy released when the envelope material recombines, is the extra energy needed to expel the CE (e.g., Han, Podsiadlowski, \& Eggleton 1994; Dewi \& Tauris 2000; Maxted et al. 2002). This proposed mechanism was criticized in previous papers (Harpaz 1998; Soker 2002; Soker \& Harpaz 2003). In Soker (2002) I criticized the paper by Maxted et al. (2002) for not considering the mass lost from the envelope prior to the onset of the CE, when the system is synchronized, i.e., the giant's rotation period equals the binary orbital period, and the binary orbital shrinkage proceeds very slowly. Eggleton (2002), for example, notes that a close companion may substantially enhance mass loss rate prior to the onset of a Roch lobe overflow (RLOF), with the possibility of preventing a $\mathrm{CE}$ phase altogether.

Soker \& Harpaz (2003) criticize Han et al. (2002) for claiming that the ionization energy in the envelope is a significant factor in the $\mathrm{CE}$ evolution. Soker \& Harpaz (2003) consider the mass lost by RGB stars as they expand by a relatively large factor from the moment of synchronization to the RLOF Soker \& Harpaz then argue that Han et al. (2002) include a mass loss rate prior to the onset of the CE that is too low, and do not include the energy radiated by the accreting white dwarf companion, as well as that emitted by the core of the giant star. In a later paper Han et al. (2003) briefly refer to Soker \& Harpaz criticism, keeping the dispute alive. Since the applicability of the $\alpha_{\mathrm{CE}}$ parameter is a fundamental question in the $\mathrm{CE}$ process, and the $\mathrm{CE}$ evolution is the channel for the formation of many close binary systems, I elaborate on some questions regarding energy and angular momentum budget in the CE phase. An extended version of this paper was submitted to another refereed journal, and can be found on (astro-ph/0311168).

\section{ENERGY AND ANGULAR MOMENTUM DEPOSITION}

An appropriate indicator for the significant of the different processes is their accumulated effect. The total energy deposited by the companion as it spirals-in from initial orbital separation $a_{0}$ to $a$ is

$$
\Delta E_{\mathrm{orb}}=\frac{G M_{1} M_{2}}{2 a}\left(1-\frac{a}{a_{0}}\right),
$$

where $M_{1}$ and $M_{2}$ are the masses of the giant and companion, respectively. Let a fraction $1-\alpha_{e}$ of this energy be radiated away. The total relative energy deposition as the companion spirals-in, the energy factor, is defined as

$$
A_{E} \equiv \frac{\alpha_{e} \Delta E_{\mathrm{orb}}}{\Delta E_{\mathrm{bind}}}=\frac{\alpha_{e}}{2 B_{\mathrm{cnv}}} \frac{M_{2}}{M_{\mathrm{env}}} \frac{R_{*}}{a}\left(1-\frac{a}{a_{0}}\right) .
$$

where

$$
\Delta E_{\mathrm{bind}}=B_{\mathrm{env}} \frac{G M_{\mathrm{env}} M_{1}}{R_{*}},
$$

is the binding energy of the expelled mass. and $B_{\text {env }} \sim 5-10 ; M_{\text {cnv }}$ is the envelope mass, and $R_{*}$ is the giant stellar radius.

The orbital angular momentum deposited to the envelope as the orbit shrinks is given, for $M_{1} \gg M_{2}$, by

$$
\Delta J_{o}=\left(G M_{1} a_{0}\right)^{1 / 2} M_{2}\left[1-\left(\frac{a}{a_{0}}\right)^{1 / 2}\right] .
$$

I assume that angular momentum deposition starts with tidal interaction, when $a_{0} \sim 4 R_{*}$. and use this value for $a_{0}$. The total angular momentum deposition factor is defined by

$$
A_{J} \equiv \frac{\Delta J_{o}}{J_{\mathrm{env}}(\max )}=\frac{1}{k_{e}} \frac{M_{2}}{M_{\mathrm{env}}}\left[1-\left(\frac{a}{a_{0}}\right)^{1 / 2}\right],
$$

where the maxinum angular momentum of the envelope, assuming a uniform rotation. is

$$
J_{\text {env }}(\max )=k_{e} M_{\text {env }}\left(G M \Lambda_{1} R_{*}\right)^{1 / 2},
$$

and $k_{e}$ is defined such that the moment of inertia of the envelope is $I_{\mathrm{env}}=k_{c} M_{\text {env }} R_{*}^{2}$, with $k_{e} \simeq 0.2$ for the considered giants.

The ratio of the angular momentum factor to the energy factor is

$$
\frac{A_{J}}{A_{E}}=100 \frac{a}{R_{*}}\left[1+\left(\frac{a}{a_{0}}\right)^{1 / 2}\right]^{-1},
$$

where the following values were used: $k_{i}=0.2, \alpha_{f}=$ 0.5 , and $B_{\text {env }}=5$.

For the parameters used to scale the last equation, it turns out that energy deposition dominates over angular momentum deposition only when $a \leqslant$ $0.01 R_{*}$. For a giant of $R_{*} \sim 1 \mathrm{AU}$, this occurs when $a \sim 2 R_{i .}$. By then many companions will go through a RLOF process. My conclusion is that for the mass loss process, in most cases it is angular momentum deposition which causes large efferts. This is trut mainly in giants which have high mass loss rate. such that the rotating envelope will facilitate much higher mass loss rate, e.g., by enhancing dust formation. 


\section{SUMMARY}

The main goal of the present paper is to point to the caution onc must take in using the $\alpha_{\mathrm{CE}}$ parameter when studying CE evolution. Namely, the orbital energy deposited to the giant's envelope is not always the main effect leading, directly or indirectly, to the removal of the envelope (see first section and my paper on astro-ph/0311168). For that I considered here the deposition of mergy from the accreting companion and the deposition of orbital angular momentum to the giant's envelope. The main results can be summarized as follows.

1. When inside the envelope of a giant, a main sequence companion is unlikely to blow jets, or a collimated fast wind (CFW, i.e., less collimated jets), or it will marginally do so only when in the outer parts of the envelope.

2. A WD companion is more likely to blow jets or a CFW.

3. These jets, ev']l if exist, whether from a WD or a MS companion, are not likely to exit the envelope at a high speed during the CE phase. Hence, they are not likely to play a major role in shaping the circumbinary matter. Jets might be blown by the companion before entering the CE (Soker \& Rappaport 2000), or by one or two of the stars after the CE ends (Soker \& Livio 1994). This explains the observations that PNs with binary nuclei are not bipolar PNs, i.e., they have no lobes, beside NGC, 2346, with the longest known orbital period. I do expect that some binary progenitors of bipolar PNs entered the CE phase at late stages, and that now the orbital separation is $\sim 0.1-1 \mathrm{AU}$. These systems are hard to detect. (Bond 2000). To obtain a quantitative result, the CE population synthesis calculations of Yungelson, Tutukov. \& Livio (1993) should be repeated but with enhanced mass loss rate from rotating AGB stars included.

4. The CFW or jets, if they exist, may inflate a bubble (with a complicated structure because of the orbital motion), hence playing a significant role in expelling the outer layers of the envelope when the companion is still orbiting in the outer envelope region.

5. In many cases the effects due to angular momentum deposition into the envelope seem more influential in removing the envelope than orbital energy deposition, assuming that fast rotating envelopes have high mass loss rates. This is true for stellar as well as substellar companions. The energy source is the giant luminosity due to nuclear energy production in the core. 'The Eddington luminosity of an accreting stellar companion is of the order of the giant's luminosity, and can farther increase the mass loss rate (Iben \& Livio 1993; Armitage \& Livio 2000).

6. My results here iterate earlier claims (Soker 2002; Soker \& Harapz 2003) that a high degree of cautious should be taken when applying the $\alpha_{\mathrm{CE}}$ parameter for the removal of CEs. For example, the conclusions of some papers that another energy source, e.g., ionization energy of the envelope, is required to remove the envelope (see criticism in Soker \& Harpaz 2003) are questionable.

I thank Mario Livio for very helpful and detailed comments at the beginning of this project. This research was partially supported by the Israel Science Foundation.

\section{REFERENCES}

Armitage, P. J., \& Livio, M. 2000, ApJ, 532, 540

Bond, H. 2000, in ASP Conf. Ser. Vol. 199, "Asymmetrical Planetary Nebulae II: From Origins to Microstructures", eds. Kastner, J. H., Soker, N., Rappaport, S. (San Francisc: Astron. Soc. Pac.) 115

Dewi, J. D. M., \& Tauris, T. M. 2000, A\&A, 360, 1043

Eggleton, P. P. 2002, ApJ, 575, 1037

Han, Z., Podsiadlowski, Ph., \& Eggleton, P. P. 1994, MNRAS, 270, 121

Han, Z., Podsiadlowski, Ph., Maxted, P. F. L., \& Marsh, T. R., 2003, MNRAS, 341, 669

Han, Z., et al., 2002, MNRAS, 336, 449

Harpaz, A. 1998, ApJ, 498, 293

Iben, I. Jr., \& Livio, M. 1993,d PASP, 105, 1373

Livio, M., \& Soker, N. 1988, ApJ, 329, 764

Maxted, P. F. L., Burleigh, M. R., Marsh, T. R., \& Bannister, N. P. 2002, MNRAS, 334, 833

O'Brien, M. S., Bond, H. E., \& Sion, E. M. 2001, ApJ, 563,971

Sandquist, E. L., Taam, R. E., \& Burkert, A. 2000 ApJ, 533,984

Soker, N. 2002, 336, 1229

Soker, N. \& Harpaz, A. 2003, MNRAS, 310, 1158

Soker, N.. \& Livio, M. 1994, ApJ, 421, 219

Soker, N.. \& Rappaport, S. 2000, ApJ, 538, 241

Taam, R. E., \& Sandquist, E. L. 2000, ARA\&A, 38, 113

Yungelson, L. R., Tutukov, A. V., \& Livio, M. 1993, ApJ, 418. 794 